\title{
Equilibrium-Based Movement Endpoints Elicited from Primary Motor Cortex Using Repetitive Microstimulation
}

\author{
Gustaf M. Van Acker, III, ${ }^{1}$ Sommer L. Amundsen, ${ }^{2}$ William G. Messamore, ${ }^{1}$ Hongyu Y. Zhang, ${ }^{1}$ Carl W. Luchies, ${ }^{2,3}$ \\ and Paul D. Cheney ${ }^{1}$ \\ ${ }^{1}$ University of Kansas Medical Center, Department of Molecular and Integrative Physiology, Kansas City, Kansas 66160, ${ }^{2}$ University of Kansas, \\ Bioengineering Graduate Program, Lawrence, Kansas 66045, and ${ }^{3}$ University of Kansas, Department of Mechanical Engineering, Lawrence, Kansas 66045
}

\begin{abstract}
High-frequency, long-duration intracortical microstimulation (HFLD-ICMS) is increasingly being used to deduce how the brain encodes coordinated muscle activity and movement. However, the full movement repertoire that can be elicited from the forelimb representation of primary motor cortex (M1) using this method has not been systematically determined. Our goal was to acquire a comprehensive M1 forelimb representational map of movement endpoints elicited with HFLD-ICMS, using stimulus parameters optimal for evoking stable forelimb spatial endpoints. The data reveal a 3D forelimb movement endpoint workspace that is represented in a patchwork fashion on the 2D M1 cortical surface. Although cortical maps of movement endpoints appear quite disorderly with respect to movement space, we show that the endpoint locations in the workspace evoked with HFLD-ICMS of two adjacent cortical points are closer together than would be expected if the organization were random. Although there were few obvious consistencies in the endpoint maps across the two monkeys tested, one notable exception was endpoints bringing the hand to the mouth, which was located at the boundary between the hand and face representation. Endpoints at the extremes of the monkey's workspace and locations above the head were largely absent. Our movement endpoints are best explained as resulting from coactivation of agonist and antagonist muscles driving the joints toward equilibrium positions determined by the length-tension relationships of the muscles.
\end{abstract}

Key words: corticomotor mapping; corticospinal; electromyography; ICMS; intracortical microstimulation; primary motor cortex

\section{Introduction}

A fundamental question in understanding cortical control of movement is how the brain uses corticomotor circuitry to coordinate complex muscle activity and movement. Corticomuscle connectivity between primary motor cortex (M1) neurons and corresponding muscles has been mapped extensively using various electrophysiological techniques. These techniques have ranged from revealing the transient increases in motor unit firing probability associated with spikes of single cortical cells or focal clusters of cells activated by single microstimuli (Cheney and Fetz, 1980, 1985; Lemon et al., 1986; McKiernan et al., 2000; Park et al., 2001), to visible twitch-like movements associated with short trains of high-frequency microstimuli (Asanuma and Sakata, 1967; Asanuma and Rosén, 1972). Data from these approaches have been widely used to construct maps of the motor

Received Jan. 16, 2014; revised 0ct. 6, 2014; accepted 0ct. 7, 2014.

Author contributions: G.M.V.A., S.L.A., C.W.L., and P.D.C. designed research; G.M.V.A., S.L.A., W.G.M., and H.Y.Z. performed research; G.M.V.A. and S.L.A. analyzed data; G.M.V.A. and P.D.C. wrote the paper.

This work was supported by National Institutes of Health Grants NS051825 and NS064054, National Institutes of Health Center Grant HD02528, the Kathleen M. Osborn Endowment, National Science Foundation Graduate STEM Fellowship in K-12 Education, and the Madison and Lila Self Graduate Fellowship. We thank lan Edwards for technical assistance.

The authors declare no competing financial interests.

Correspondence should be addressed to Dr. Paul D. Cheney, University of Kansas Medical Center, Department of Molecular \& Integrative Physiology, 3901 Rainbow Blvd, Mail Stop 3043, Kansas City, KS 66160-7336. E-mail: pcheney@kumc.edu.

DOI:10.1523/JNEUROSCI.0214-14.2014

Copyright $\odot 2014$ the authors $\quad 0270-6474 / 14 / 3415722-13 \$ 15.00 / 0$ cortical representation of movements and muscles. For example, previous work from our laboratory established consistent and reproducible intra-areal features of the forelimb cortical representation with separate zones for distal and proximal muscles as well as a zone producing activation of combinations of distal and proximal muscles (Park et al., 2001).

An alternative to applying short trains of high-frequency stimuli to elicit twitch-like movements in the periphery, which reveals only the joints involved, is to apply high-frequency, longduration intracortical microstimulation (HFLD-ICMS), which elicits complete movements to endpoints in space that resemble natural movements (Graziano et al., 2002). Graziano et al. (2002) reported an important and fundamental feature of these movements, namely, that stimulation of a given cortical site produces movement to the same endpoint in the workspace regardless of the initial location of the hand.

Recently, we established stimulation parameters yielding HFLD-ICMS movements to stable movement endpoints (Van Acker et al., 2013). The goal of the present study was to use these parameters to systematically map evoked movement endpoints over the full extent of forelimb M1 cortex with the intent of revealing possible orderly features in maps of endpoint locations. The results reveal a relatively extensive M1 stimulus-evoked movement repertoire, although representing primarily equilibrium-based spatial endpoints as opposed to the full range of possible endpoints based on the physical capability of the forelimb. The representation of endpoint positions is a patchwork in 
which clusters of adjacent cortical sites often represent adjacent endpoint locations in the $3 \mathrm{D}$ workspace. However, although the overall representation of cortical endpoint locations across M1 cortex is not random, the relative disorder of the representation upon visual inspection is most apparent.

\section{Materials and Methods}

Behavioral task. Data were collected from two male rhesus macaques (Macaca mulatta; $\sim 9 \mathrm{~kg}$ each, ages 4 and 12 years) performing a reach and prehension task that required coactivation of proximal and distal forelimb muscles while also yielding discrete and repeatable spatial locations at which stimulus trains could be cued. Training procedures and the behavioral task have been described in detail previously (Belhaj-Saiff et al., 1998; McKiernan et al., 1998). Briefly, each monkey was seated in a custom primate chair within a sound-attenuating chamber with his left arm restrained while his right arm retained freedom of movement. To discourage the monkey from interfering with reflective motion capture spheres attached to his arm, during each recording session a custom face-mask barrier was installed on the chair that contained a small hole through which the monkey could feed himself. In addition, a waist plate prevented the monkey from interfering with the spheres using his feet. To receive a food reward, the monkey initiated the task with his right hand by depressing a lever located at waist height for $1 \mathrm{~s}$ duration, which triggered the release of a food pellet into a food well located approximately at shoulder height requiring full arm extension for retrieval. Performance was guided by audio and visual cues.

Surgical procedures. After training, an MRI-compatible stainless steel chamber allowing $30 \mathrm{~mm}$ diameter of dura exposure and access to the underlying cortical area was implanted stereotaxically over M1 of the left hemisphere of each monkey using procedures described in detail previously (Park et al., 2000). Briefly, the chamber was anchored to the skull using titanium screws and dental acrylic, and was centered over the hand area of M1 in the left hemisphere. In addition, threaded titanium nuts were attached to the occipital aspect of the skull using titanium screws and dental acrylic. These nuts provided a point of attachment for a flexible head restraint during data collection (McKiernan et al., 1998, 2000). The chambers were centered at anterior $17.7 \mathrm{~mm}$, lateral $27.2 \mathrm{~mm}$, and $26^{\circ}$ to the sagittal plane for Monkey X, and anterior $16.0 \mathrm{~mm}$, lateral 22.0 $\mathrm{mm}$, and $30^{\circ}$ to the sagittal plane for Monkey A.

Twenty-four muscles of the right shoulder and forelimb were each implanted with two multistranded stainless steel wires (Cooner Wire, AS632). Both monkeys were implanted under sterile conditions using a cranial subcutaneous implant technique described previously (Park et al., 2000). Briefly, all wires were stripped of $\sim 2-3 \mathrm{~mm}$ of insulation and tunneled subcutaneously from a cortical connector (Amphenol) to each muscle, where the wires were inserted into the muscles a distance of $\sim 2-3 \mathrm{~cm}$, with a $\sim 5 \mathrm{~mm}$ separation of the two wires in each muscle. The cortical connector module was secured with dental acrylic near the cortical recording chamber. We tested the placement accuracy of each electrode pair by observing appropriate muscle twitches that resulted from applying short stimulus trains (Grass SD9 Stimulator). EMG activity was recorded from five shoulder muscles: pectoralis major, anterior deltoid, posterior deltoid, teres major, and latissimus dorsi; seven elbow muscles: biceps short head, biceps long head, brachialis, brachioradialis, triceps long head, triceps lateral head, and dorsoepitrochlearis; five wrist muscles: extensor carpi radialis, extensor carpi ulnaris, flexor carpi radialis, flexor carpi ulnaris, and palmaris longus; five digit muscles: extensor digitorum communis, extensor digitorum 2 and 3, extensor digitorum 4 and 5, flexor digitorum superficialis, and flexor digitorum profundus; and two intrinsic hand muscles: abductor pollicis brevis and first dorsal interosseus.

All surgeries were performed under general anesthesia and aseptic conditions. Before surgery, the monkey was administered ketamine (10 $\mathrm{mg} / \mathrm{kg}$, i.m.), atropine (0.04 mg/kg, i.m.), and medetomidine $(0.05 \mathrm{mg} /$ $\mathrm{kg}$, i.m.) for transportation purposes, and subsequently isoflurane gas for the duration of the surgery. Each monkey received prophylactic antibiotic (penicillin, $6000 \mathrm{U} / \mathrm{kg}$ ) subcutaneously $10 \mathrm{~h}$ before surgery, $1 \mathrm{~h}$ after surgery, and $3 \mathrm{~d}$ after surgery. Postoperatively, each monkey was given analgesics (buprenorphine, $0.01 \mathrm{mg} / \mathrm{kg}$ i.m. and carprofen, $5 \mathrm{mg} / \mathrm{kg}$ s.c.). All surgeries were performed in a facility accredited by the Association for Assessment and Accreditation of Laboratory Animal Care using full sterile procedures. All procedures were approved by the Institutional Animal Care and Use Committee and conformed to the Guide for the Care and Use of Laboratory Animals, published by the United States Department of Health and Human Services and the National Institutes of Health.

Stimulus-triggered averages (StTAs) and determining basic output maps. StTAs were acquired for all implanted muscles from stimuli applied throughout all phases of the reach and prehension task and were used to help confirm electrode positioning as well as to determine corticomuscle connectivity of corticospinal cells directly surrounding the electrode tip. Stimulation was applied through glass insulated platinum-iridium electrodes suitable for single-unit recording (FHC), with typical impedances of $0.7-1.5 \mathrm{M} \Omega$ at the start of each recording session. We positioned the electrodes using an $\mathrm{X}-\mathrm{Y}$ positioner secured to the chamber for the duration of each recording session, and advanced the electrode into the brain using a manual hydraulic microdrive (FHC) until the electrode tip was located putatively in cortical lamina $\mathrm{V}, \sim 1.5 \mathrm{~mm}$ below the surface of the brain. Location of layer $\mathrm{V}$ was determined by depth, audio cues (Grass AM8 Audio Monitor), visual cues (oscilloscope display of large spike waveforms), and finally by poststimulus effects (PStEs) in StTAs at $15 \mu \mathrm{A}$ and $10 \mathrm{~Hz}$.

StTAs were obtained at $10 \mathrm{~Hz}$ and current intensities that matched those used for each HFLD-ICMS train applied at the same cortical site. In addition, muscle facilitation maps (see Fig. $1 A, B$ ) used StTAs collected at 15 and $30 \mu \mathrm{A}$ to plot the underlying representations of distal, proximal, and proximal-distal muscle representations (Park et al., 2001). Mapping was done at $1 \mathrm{~mm}$ intervals on the surface of the precentral gyrus and 0.5 $\mathrm{mm}$ vertical intervals down the bank of the central sulcus as described previously (Park et al., 2001). Determining boundaries of M1 ensured complete HFLD-ICMS mapping of the M1 forelimb representation. Criteria for identifying the borders of M1 with PMd and PMv rostrally were the loss of effects and/or an increase in PStE threshold, an increase in stimulus threshold for eliciting movements, and/or the finding of trunk movements rostromedially and face movements rostrolaterally. These are similar to criteria previously described (Stepniewska et al., 2006). EMG activity was filtered from $30 \mathrm{~Hz}$ to $1 \mathrm{kHz}$, digitized at $4 \mathrm{kHz}$, and full-wave rectified. Individual stimuli for the StTAs were symmetrical biphasic pulses: $0.2 \mathrm{~ms}$ negative pulse followed by a $0.2 \mathrm{~ms}$ positive pulse. StTAs were based on a minimum of 1000 trigger events. Averages were compiled using a $60 \mathrm{~ms}$ epoch, of which $20 \mathrm{~ms}$ before the trigger was considered baseline. StTAs were identified as having significant PStEs if the peak or trough of the effect exceeded $\pm 5 \mathrm{SD}$ of the baseline for a period of $\geq 0.75 \mathrm{~ms}$ as described previously (Park et al., 2004).

HFLD-ICMS protocol. HFLD-ICMS trains were applied to 251 distinct cortical sites (see Fig. 1 $A, B$ ) over the course of the study in the left hemispheres of two rhesus macaques performing a reach and prehension task. Stimulation was applied when the monkey's hand was at one of three positions of the behavioral task (home-plate, feeder, mouth). A stimulus onset location that was furthest from the stimulus-evoked spatial endpoint was chosen once a test trial elicited a detectable movement. Because the feeder was on the periphery of the monkey's workspace requiring full arm extension, this starting location for stimulation was used most frequently. Starting location for stimulation had no noticeable effect on the spatial endpoints elicited in the present study, consistent with previous studies (Graziano et al., 2002, 2005).

Cortical sites within M1 of each monkey were mapped in random order at a resolution of $1 \mathrm{~mm}^{2}$ on the surface of the gyrus and $0.5 \mathrm{~mm}$ along descending tracks in the rostral bank of the central sulcus. We applied systematic combinations of frequency and stimulus intensity with a stimulus train duration of $1 \mathrm{~s}$ until either a combination of parameters was qualitatively assessed visually to yield a successful spatial endpoint, or it was determined that no spatial endpoint could be elicited. Sites that did not yield spatial endpoints yielded either minimal movement upon stimulation, or affected distal or proximal muscles independently, thereby allowing voluntary movement of the unaffected muscles during stimulation consequently yielding unstable movement endpoints. 
Data from previous work in our laboratory (Van Acker et al., 2013) demonstrated that the median successful parameters that elicited translocation with subsequent stabilization of the forelimb at spatial endpoints were $110 \mathrm{~Hz}, 110 \mu \mathrm{A}$, and a stimulus train duration of $1 \mathrm{~s}$. These parameters were used for the majority of the trials that yielded successful endpoints achieved throughout the present study $(63.4 \%$ and $75.0 \%$ for Monkey A and Monkey X, respectively), with the remaining sites requiring stimulation parameters in the ranges of $90-150 \mathrm{~Hz}$ and $90-150 \mu \mathrm{A}$. For each HFLD-ICMS parameter set, we conducted five trials. Real-time qualitative assessment of successful spatial endpoints for each site were determined by subjective visual evaluation. Kinematic data were later used in offline analyses to quantitatively map successful spatial endpoints.

The stimulus parameters used, although high, did not appear to produce tissue damage surrounding the site of stimulation. We applied biphasic charge-balanced pulses to minimize the possibility of tissue lesion resulting from stimulation. Additionally, cortical sites receiving stimulation remained sensitive to repeated stimulation, for both intrasession and intersession stimulation repetitions. Also, we often could record cell spikes after stimulation.

Recording forelimb kinematics. Limb kinematics were monitored and recorded using a Vicon motion capture system. For data collected in the present study, a virtual sphere located on the dorsal tubercle of the radius was calculated and used to evaluate HFLD-ICMS-evoked forelimb kinematics and the spatial endpoint positions elicited from each cortical site. The 3D coordinates of this virtual sphere were calculated using a modified Point Cluster technique similar to that described previously (Andriacchi et al., 1998; Senesh and Wolf, 2009). Briefly, a cluster of 10 reflective spheres were attached to the forearm of each monkey. The 3D coordinates of the spheres in each cluster, as measured using the Vicon system, were used to triangulate the coordinates of the virtual sphere located on the dorsal tubercle of the radius, which was identified during static trials at the start of each recording session. The dorsal tubercle of the radius was used as a surrogate for endpoint position of the limb in space. Using subsets of the numerous wrist markers to calculate the spatial position of the reference point (dorsal tubercle of the radius) reduced the need for the wrist to be within view of any particular set of Vicon cameras to be monitored. The monkey's workspace was represented as a cube subdivided into 27 voxels. Each voxel $(154.2 \times 126.1 \times 98.7 \mathrm{~mm})$ was colorcoded to enable visualization of subdivisions within the monkey's workspace (see Fig. 2A).

Determining successful spatial endpoints for HFLD-ICMS-evoked movements. HFLD-ICMS-evoked kinematics of the dorsal tubercle of the radius were used to determine successful spatial endpoints of the wrist achieved through M1 stimulation. HFLD-ICMS-evoked movement trials were deemed successful if the stimulus parameters applied to the M1 site were sufficient to translocate the monkey's forelimb to a spatial location distinct from its location at the initiation of stimulation and sufficient to maintain the limb at the stimulus-evoked posture for the duration of stimulation. The specific success criteria we used required, first, that stimulus-evoked movement velocity reach a level of $\geq 40 \%$ of the average maximum stimulus-evoked velocity within the first $500 \mathrm{~ms}$ of stimulation onset to indicate adequate stimulus-induced movement. The average maximum stimulus-induced velocity was calculated from a subset of $\sim 25 \%$ of the data for which we determined success based upon individual trial velocity data. This iterative process allowed for final analysis of all trials using consistent velocity thresholds. The second criterion was that, on the slowing phase of movement, the velocity had to cross below a threshold of $25 \%$ of the average maximum stimulus-induced velocity. Finally, once this lower threshold was crossed, the velocity of the movement had to remain below this $25 \%$ threshold for the remainder of the applied stimulus train to ensure a stable spatial endpoint had been achieved. Spatial endpoint position was measured at the point where the velocity of the stimulus-evoked movement crossed the threshold of $25 \%$ toward achieving a stable endpoint position. These objective endpoints closely matched endpoints identified using real-time subjective visual assessment.

Measurement of EMG cross talk. We evaluated cross talk between EMG electrodes by constructing EMG-triggered averages. This procedure used the motor unit potentials from one muscle as triggers for compiling averages of rectified EMG activity of all other muscles. Criteria established previously (Buys et al., 1986) were used to eliminate effects that might have been affected by cross talk. To be accepted as a valid PStE, the ratio of poststimulus facilitation between the test and trigger muscles must have exceeded the ratio of their cross talk peaks by a factor of $\geq 2$. Based on this criterion, first dorsal interosseus in Monkey X was removed from the analysis.

\section{Results}

\section{HFLD-ICMS-evoked movement relative to M1 forelimb representation}

We applied HFLD-ICMS systematically to individual cortical sites throughout the entire M1 forelimb representation (Fig. $1 A, B)$ while the monkey performed a reach and prehension task, with the goal of obtaining a comprehensive map of stimulusevoked forelimb endpoint positions represented therein. Each monkey's M1 forelimb representation was characterized by assessing corticomuscle connectivity of the right forelimb as transient increases in EMG activity via StTA (Cheney and Fetz, 1985) and are consistent with forelimb muscle facilitation maps reported previously (Park et al., 2001). This corticomuscle connectivity is represented by the color-coded background in the maps of Figure 1. To evoke stable movement endpoints, at each cortical site, we started with stimulation parameters (typically $110 \mathrm{~Hz}$, $110 \mu \mathrm{A}$, and $1 \mathrm{~s}$ duration) determined previously in our laboratory to be effective and safe parameters for evoking successful forelimb movement to stable spatial endpoint positions in an awake monkey (Van Acker et al., 2013). If successful stimulusevoked movements were not obtained, stimulus parameters were adjusted until a clear spatial endpoint was achieved or until we determined that no stable endpoint could be obtained. We concluded that forelimb endpoints were not inducible if sufficient movement was not evoked at stimulation parameters up to 150 $\mathrm{Hz}$ and $150 \mu \mathrm{A}$, and/or stimulation affected only proximal or only distal muscles, which allowed the monkey to voluntarily move unaffected muscles and associated joints during stimulation. For all cortical sites in the present study, the range of parameters used to evoke a stable forelimb endpoint posture was a frequency of $90-150 \mathrm{~Hz}$ and a stimulus intensity of $90-150 \mu \mathrm{A}$. The combination of $110 \mathrm{~Hz}$ and $110 \mu \mathrm{A}$ produced successful movements and stable endpoints in $63.4 \%$ and $75.0 \%$ of the successful trials in Monkey A and Monkey X, respectively.

The monkey's workspace is represented as color-coded voxels, illustrated in Figure $2 A$ as anterior-posterior, mediolateral, and superior-inferior views to enable visualization of spatial endpoints (Fig. 2B) evoked from corresponding cortical sites. Movement kinematics and spatial endpoint positions of a virtual sphere located on the wrist (dorsal tubercle of the radius) were calculated and used to determine HFLD-ICMS-evoked spatial endpoint positions elicited from each cortical site. Spatial endpoints in Figure $2 B$ have numbers corresponding to stimulation sites in the cortical maps of Figure 1. The monkey's workspace is divided into lateral, central, and medial color-coded planes from a mediolateral perspective for both Monkey A (left) and Monkey $\mathrm{X}$ (right). The similarity between the spectrum of endpoint distributions that were elicited from M1 forelimb representations in both monkeys is clearly evident when subdivided into these mediolateral spatial planes. Endpoints were preferentially located in the central plane with fewer sites in the medial and lateral planes consistent with the work of Graziano et al. (2002). There was also a relatively limited number of endpoints in upper space, again consistent with Graziano et al. (2002).

Figure 3 documents the variability of spatial endpoints achieved from stimulation at individual cortical sites. Figure $3 A$ shows a sin- 

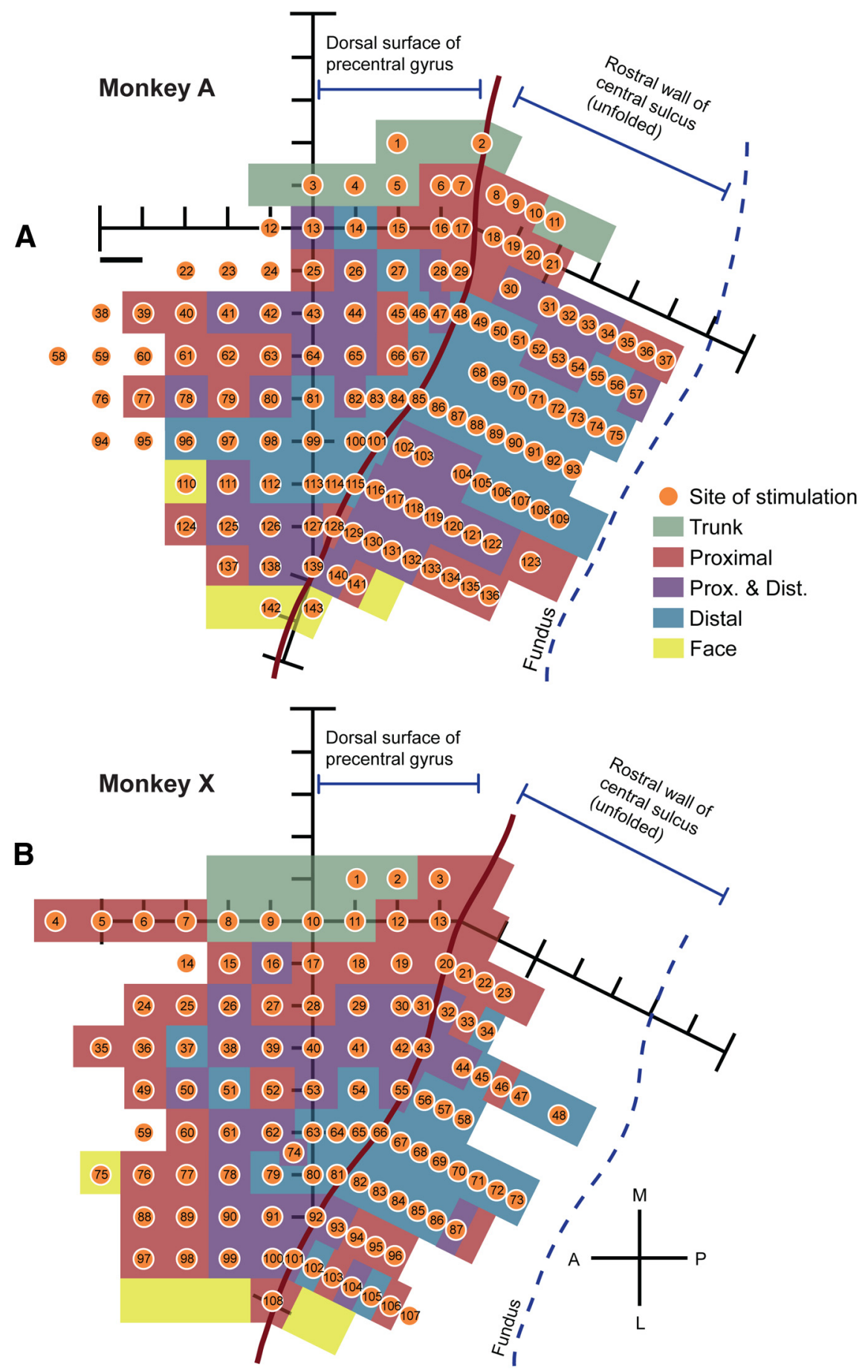

Figure 1. Maps of stimulation sites in two monkeys ( $\boldsymbol{A}$, Monkey $A ; \boldsymbol{B}$, Monkey $X)$ from which data were collected. HFLD-ICMSmapped sites are indicated by the numbered orange dots. The rostral wall of the central sulcus is unfolded into the same $2 D$ plane as the dorsal surface of the precentral gyrus. The compass rose represents anterior, posterior, medial, and lateral. HFLD-ICMSmapped sites are numbered in logical order for presentation purposes rather than chronological order of data collection. Areas producing muscle facilitation in StTAs of distal forelimb muscles (blue), proximal forelimb muscles (red), and both proximal and distal muscles (purple) are indicated. Scale bar, $1 \mathrm{~mm}$.

gle cortical site example of the stimulus-evoked movement trajectories to spatial endpoints from multiple starting locations for each monkey (Monkey A in the left column, Monkey X in the right column). Open circles represent the start of stimulation; closed circles represent the end. For Monkey A, traces showing stimulation initiation when the hand was at the home-plate (purple), mouth (black), and food well (remaining colors) are shown, with the trajectories ending near similar spatial endpoints. For Monkey X, traces show stimulation initiated when the hand was midway between the home-plate and food well (green) and at the food well (remaining colors), with trajectories converging on similar endpoints. Figure $3 B$ shows histograms categorizing the average spatial distribution of endpoints elicited from each cortical site. Mean spatial variability for each cortical site was calculated as the mean of the $x, y$, and $z$ ranges across endpoints at each cortical site. For example, if four endpoints were achieved from one cortical site, the maximum and minimum values of the $x, y$, and $z$ coordinates were determined and the mean of those three ranges was calculated as the mean spatial variability for that site. Sixteen sites for Monkey A and 11 sites for Monkey X yielded only one endpoint from which variability could not be calculated. Although spatial endpoint ranges for cortical sites were variable, most had average endpoint spreads $<50 \mathrm{~mm}$ for each monkey.

Cortical representation maps of stimulation-evoked forelimb spatial endpoints elicited from each cortical site were constructed (Fig. 4A,B), with the color at each site corresponding to a portion of the monkey's workspace represented as color-coded voxels in Figure 2A. The endpoint coordinates for each site were the mean coordinates calculated from all successful stimulation-evoked movement trials elicited from that site. We found that, although maps of cortical sites yielding successful HFLD-ICMS evoked stable spatial endpoints (Fig. 4A,B) tended to be located within or near maps of cortical output zones that produced StTA-derived facilitation of both proximal and distal muscles (purple cortex, Fig. $1 A, B$ ), the two maps did not overlap precisely. This likely reflects the use of higher stimulus intensities with HFLD-ICMS (90-150 $\mu \mathrm{A})$ compared with the intensities applied during StTA mapping $(15-30 \mu \mathrm{A})$, resulting in activation of a greater number of corticospinal neurons (Tehovnik et al., 2006) targeting a larger muscle ensemble. In addition, the higher stimulus frequencies used with HFLD-ICMS likely resulted in greater physiological spread (Jankowska et al., 1975) through corticocortical connections, further eroding the muscle facilitation boundaries defined with StTA. Some cortical sites yielding clear PStEs in both proximal and distal muscles did not yield stable spatial endpoints (Fig. 4A, black sites). Although they did not yield stable endpoints, most of these sites did produce movement. However, nonstable movements were not quantified in this study. One possible explanation for unachievable steady-state 


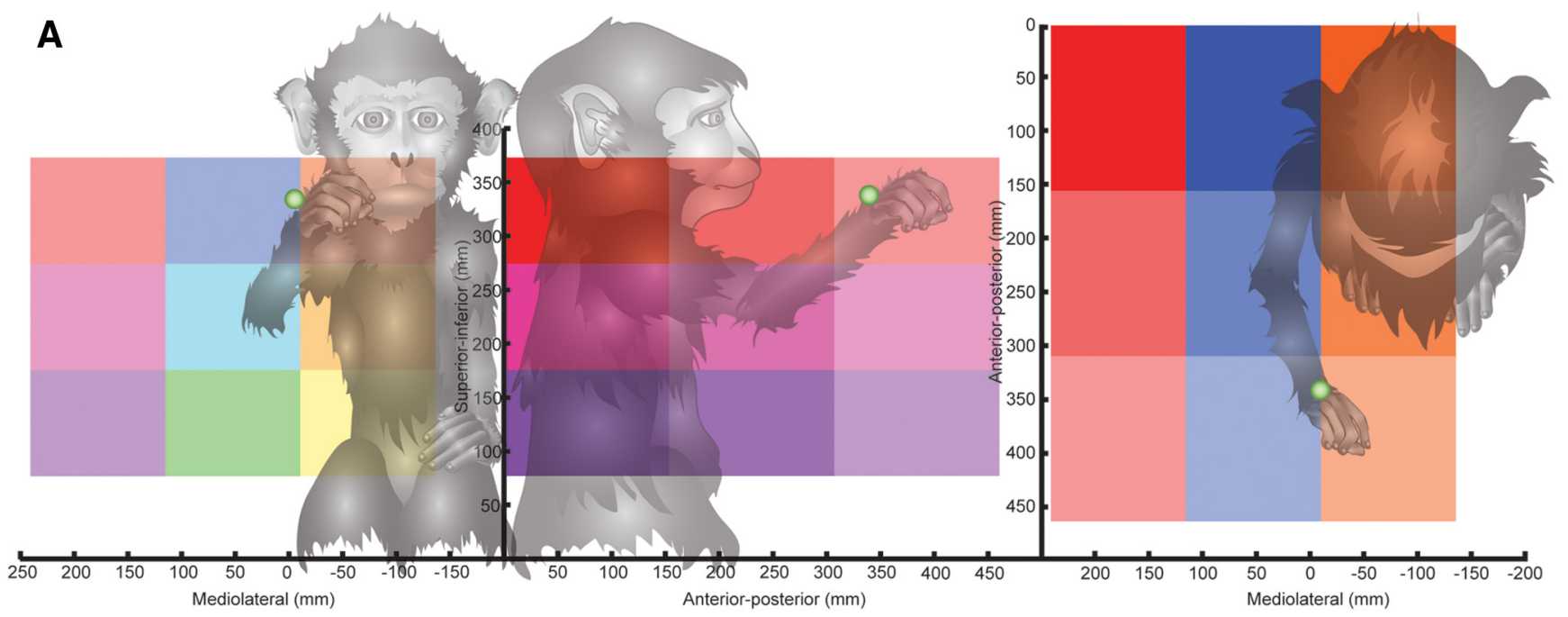

B
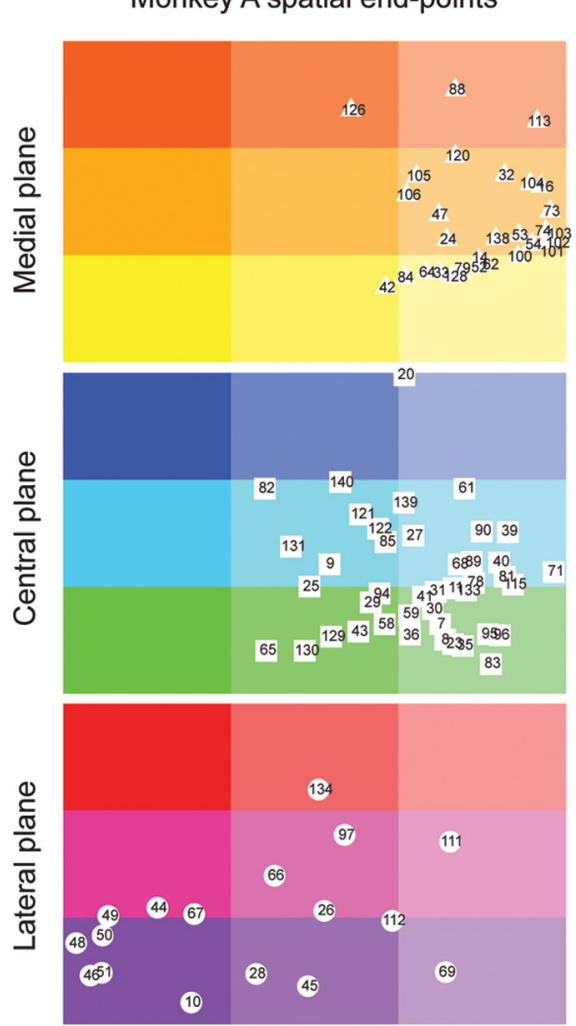

Monkey X spatial end-points
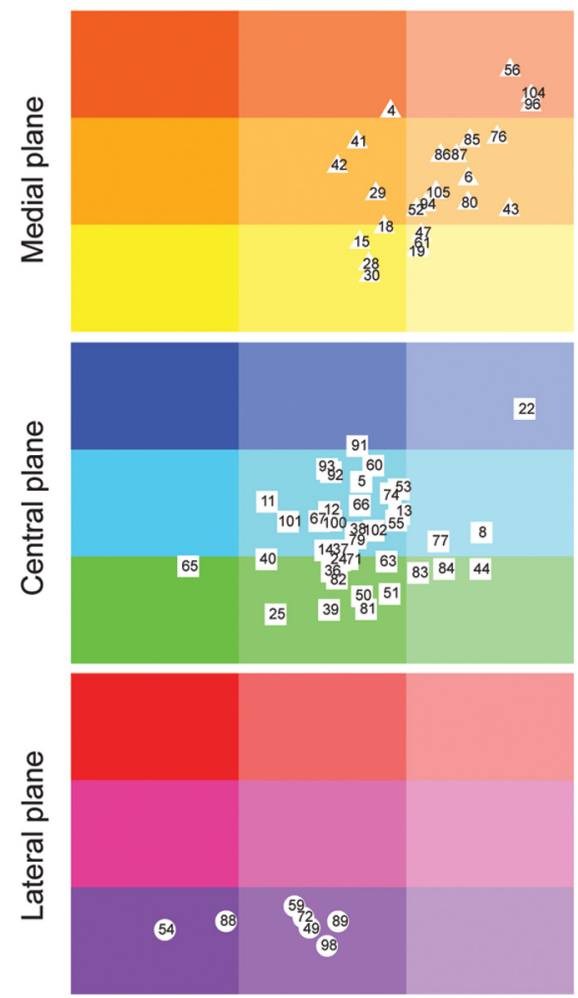

Figure 2. Color-coded workspace and HFLD-ICMS-evoked movement endpoint locations. $A$, Anterior-posterior, mediolateral, and superior-inferior views of color-coded voxels representing the monkey's workspace. Coordinates measured in millimeters are indicated for the three dimensions. Monkeys were situated with midsagittal plane at approximately mediolateral coordinate -100 $\mathrm{mm}$, and mid-coronal plane at approximately anterior-posterior $100 \mathrm{~mm}$. Voxel size is $154.2 \mathrm{~mm}$ in the anterior-posterior direction, $126.1 \mathrm{~mm}$ mediolateral, and $98.7 \mathrm{~mm}$ superior-inferior. $\boldsymbol{B}$, HFLD-ICMS-evoked spatial endpoints for each cortical site, represented by numbers for Monkey $A$ and Monkey $X$ corresponding to cortical site numbers in Figure $1 A$ and Figure $1 B$, respectively. Parasagittal planes are divided into medial, central, and lateral planes with dimensions corresponding to those in the color-coded voxels.

endpoints at these locations is that stimulus-evoked activity affected a limited proportion of the proximal and distal muscles about the joints, enabling voluntary movement in remaining muscles.

\section{Extent of workspace represented by HFLD-ICMS-evoked endpoints}

The results reveal HFLD-ICMS-evoked forelimb spatial endpoints that encompass a considerable range of locations through- out the available forelimb workspace, although the vast majority are centrally oriented with respect to the torso and the physical capability of forelimb movement, with limited peripheral movements and no movements above head level (Fig. 2A). Figure $5 A, B$ represents $2 \mathrm{D}$ anterior-posterior and mediolateral views of the monkeys performing the reach and prehension task, with superimposed successful spatial endpoints elicited from each cortical site in M1 for each monkey. Included in Figure 5 are representations of the workspaces (light 
Monkey A

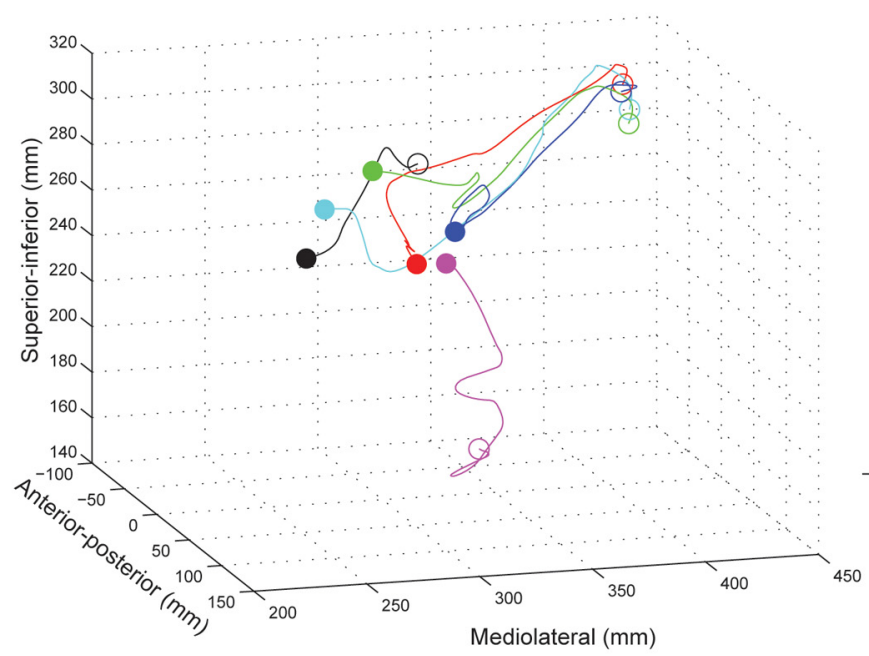

Monkey X

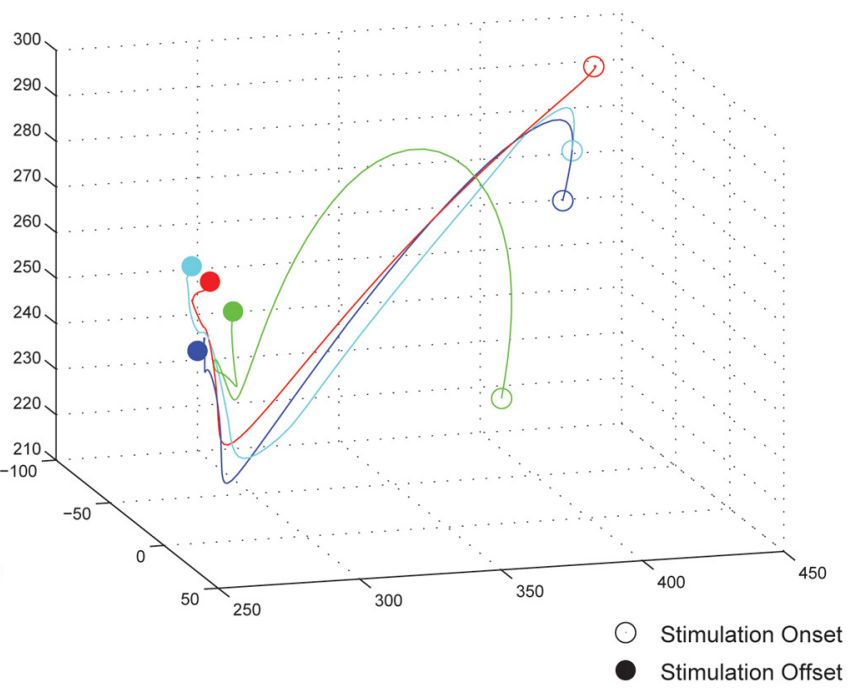

B Distribution of HFLD-ICMS-evoked end-point variability for each cortical site
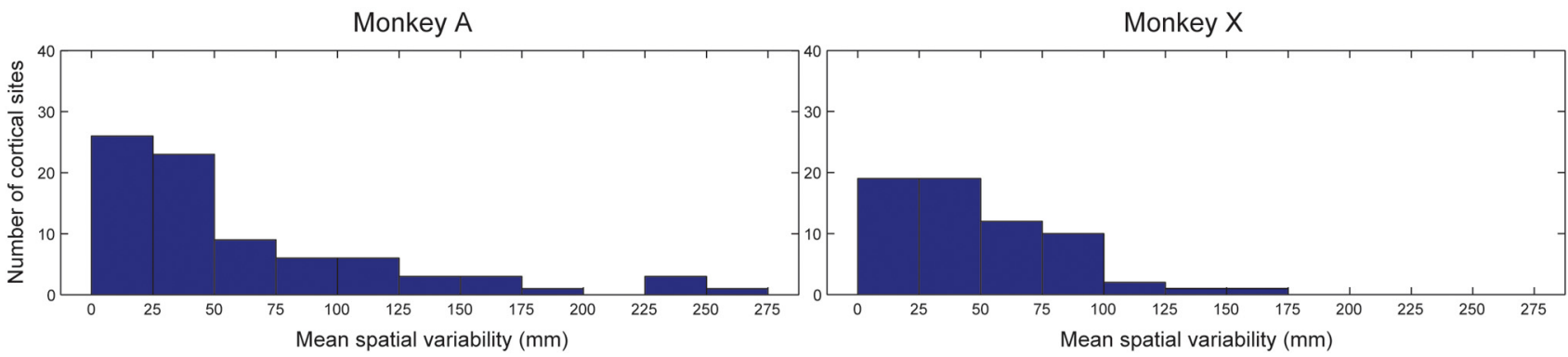

Figure 3. Distribution of endpoint variability. A, HFLD-ICMS-evoked trajectories from multiple starting locations for single cortical sites in each monkey. For both Monkey A (left column) and Monkey X (right column), stimulus-evoked movement trajectories are shown: open circles represent the start of stimulation; solid circles represent stimulation end. The stimulation time for each trajectory is $1 \mathrm{~s}$. Scale axes are different in each case. For Monkey A, traces showing stimulation initiation when the hand was at the home-plate (purple), mouth (black), and food well (remaining colors) are shown. For Monkey X, traces showing stimulation initiation when the hand was midway between the home-plate and food well (green) and at the food well (remaining colors) are shown. $B$, Histograms categorizing the mean spatial distribution of endpoints elicited from each cortical site. Mean spatial variability was calculated as the mean of the $x, y$, and $z$ ranges across endpoints at each cortical site. Sixteen sites for Monkey A and 11 sites for Monkey X yielded only one endpoint from which variability could not be calculated.

blue sphere section) through which the dorsal tubercle of the radius (green sphere) was physically capable of moving. The majority of the spatial endpoints reside on the limb's ipsilateral side of the midsagittal plane and below the level of the monkey's head, likely reflecting the fact that HFLD-ICMS activates antagonistic muscle synergies encompassing both flexors and extensors at each joint. This increases the likelihood that the resulting movement equilibrium points will end at locations other than the most peripheral points in the workspace. It should be noted that some movements were blocked from continuing to completion due to physical barriers present. While rarely impeding movement, one such barrier was a protective face shield surrounding the monkey's head. Additionally, a home-plate lever and protective waist plate (see Fig. 5 , blue sphere) prevented movement below waist level. However, it is noteworthy that there is no obvious concentration of points at the plane of the barriers, suggesting that the barriers did not significantly restrict HFLD-ICMS evoked movements.

\section{Relationship between spatial endpoints evoked from adjacent} cortical sites

Many spatial endpoints generated by stimulation of adjacent M1 cortical sites were spatially contiguous in the monkey's workspace; however, these tended to exist within aggregates relative to the entirety of M1, and most of these aggregates were adjacent to others yielding disparate endpoints. This yielded an overall cortical map of endpoint positions in which the monkey's 3D workspace was represented in a patchwork fashion on the $2 \mathrm{D}$ M1 cortical surface. This can be seen as pockets of similar spatial color coding of adjacent cortical sites in Figure $4 A, B$. To further test this relationship, Figure $6 A, B$ displays the color-coded mean 3D Euclidean spatial endpoint distances for each cortical site to the endpoint locations of all 2D adjacent cortical sites for Monkey A and Monkey X, respectively. Adjacent cortical sites where stimulation failed to evoke stable spatial endpoints were excluded from this analysis. For example, cortical site 14 in Figure $6 \mathrm{~A}$ (top yellow box) is color-coded as the mean $3 \mathrm{D}$ distance between the spatial endpoint evoked from site 14 and the spatial endpoints 
evoked from sites 25, 26, and 27. The mean 3D distance between spatial endpoints in the workspace corresponding to all combinations of adjacent cortical sites was $156.1 \pm 94.2 \mathrm{~mm}$ for Monkey A and $113.8 \pm 63.4 \mathrm{~mm}$ for Monkey $\mathrm{X}$, whereas the maximum 3D distance between any two spatial endpoints in the workspace was $520.5 \mathrm{~mm}$ for Monkey A and 421.1 $\mathrm{mm}$ for Monkey X. For reference, the mean length of each monkey's hand, from the dorsal tubercle of the radius to the tip of the third digit, was $\sim 106 \mathrm{~mm}$, and the mean length of each arm, from the greater tubercle of the shoulder to the dorsal tubercle of the radius, was $\sim 298 \mathrm{~mm}$. We also compared the mean $3 \mathrm{D}$ distance for all adjacent cortical combinations (156.1 $\pm 94.2 \mathrm{~mm}$ for Monkey A, $113.8 \pm$ $63.4 \mathrm{~mm}$ for Monkey X) to the mean 3D distance for all possible cortical site combinations other than adjacent cortical sites $(185.1 \pm 101.5$ for Monkey A, $140.5 \pm$ 70.4 for Monkey X). For both monkeys, the mean 3D distance for all adjacent cortical sites is significantly less than the mean 3D distance for all possible cortical sites without duplicates ( $p<0.001$, Man$\mathrm{n}$-Whitney). Of the adjacent cortical site combinations, 140 of $204(68.6 \%)$ of the corresponding 3D endpoint distances were below the mean of those for all cortical site pairs in Monkey A, and 117 of $164(71.3 \%)$ were below the mean for Monkey X. Finally, we computed 10 sets of randomized cortical site combinations to assess the mean 3D endpoint distance if cortical organization with respect to endpoint position were completely random. The results (183.5 \pm 88.8 for Monkey A, $140.6 \pm 71.7$ for Monkey X) are similar to the mean 3D distances for all possible cortical site combinations noted above and significantly greater than the mean distance of adjacent cortical sites.

Another way to evaluate the data in Figure 6 is to ask what the spatial separation of endpoints would be if there were a completely orderly representation of the workspace across M1 cortex. The boundary of the monkey's workspace is defined by the perimeter obtained by the path of the hand as the straightened arm is rotated in various orientations at the shoulder (Fig. 5). For our monkeys, this was $\sim 600$ $\mathrm{mm}$ in both the mediolateral and vertical directions. The arm/hand representation in $\mathrm{M} 1$ cortex occupies a cortical territory of $\sim 10 \times 10 \mathrm{~mm}$. If the workspace were represented in a completely orderly graded fashion across this cortical area, a distance of $0.5-1 \mathrm{~mm}$ in the cortex (our
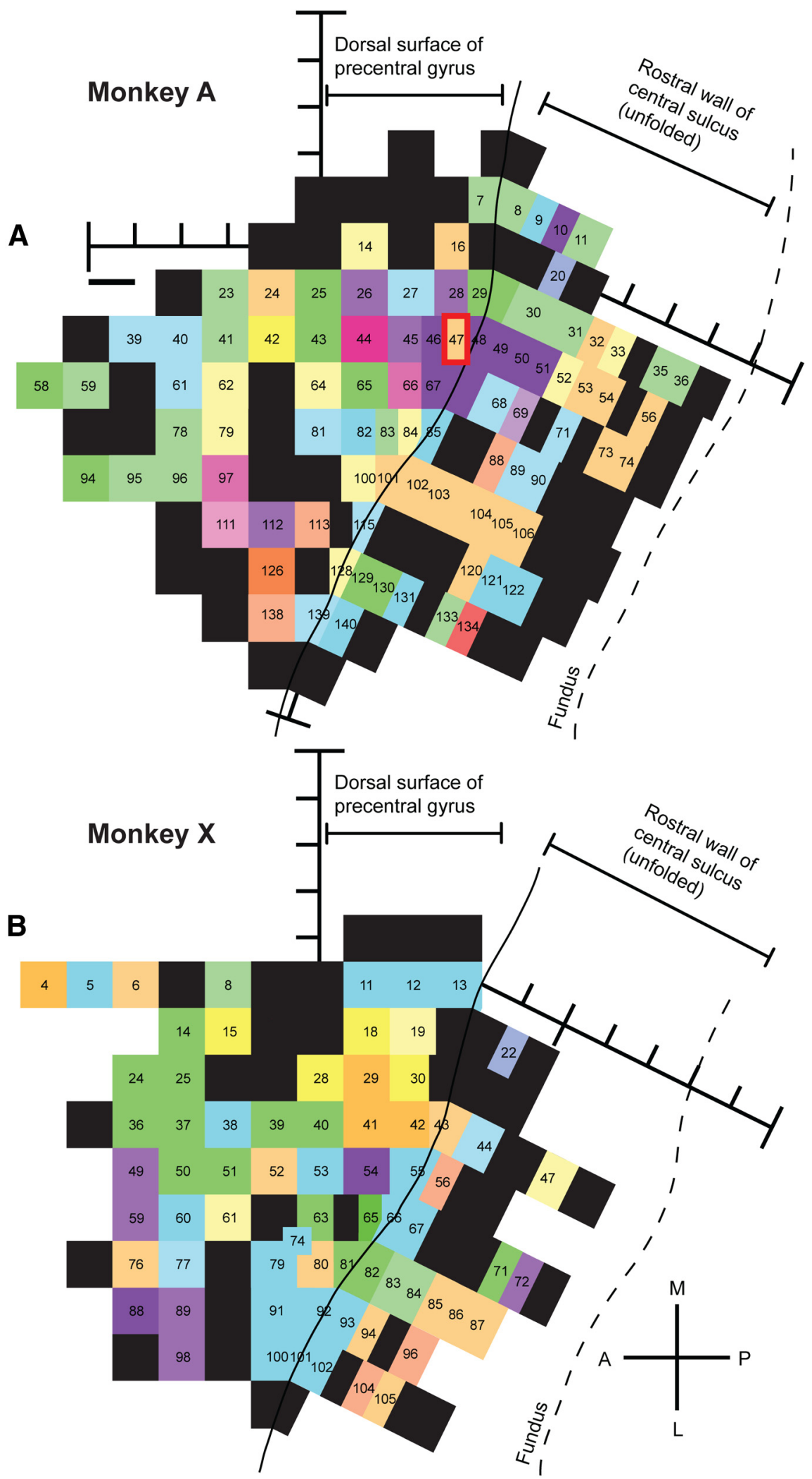

Figure 4. Cortical representation of color-coded spatial endpoints. Cortical sites for Monkey $A(A)$ and Monkey $X(B)$ are color-coded corresponding to the workspace voxels in Figure 2 used to categorize the location of the HFLD-ICMS-evoked spatial endpoints elicited from each site. Numbered cortical sites correspond to spatial endpoints represented in Figure 2B. Colored sites are those that yielded successful spatial endpoints of the forelimb with applied HFLD-ICMS, whereas black sites are those that did not produce movements to stable endpoints, although in most cases movements were evoked. The rostral wall of the central sulcus is unfolded into the same 2D plane as the dorsal surface of the precentral gyrus. Compass rose represents anterior, posterior, medial, and lateral. Scale bar, $1 \mathrm{~mm}$. 


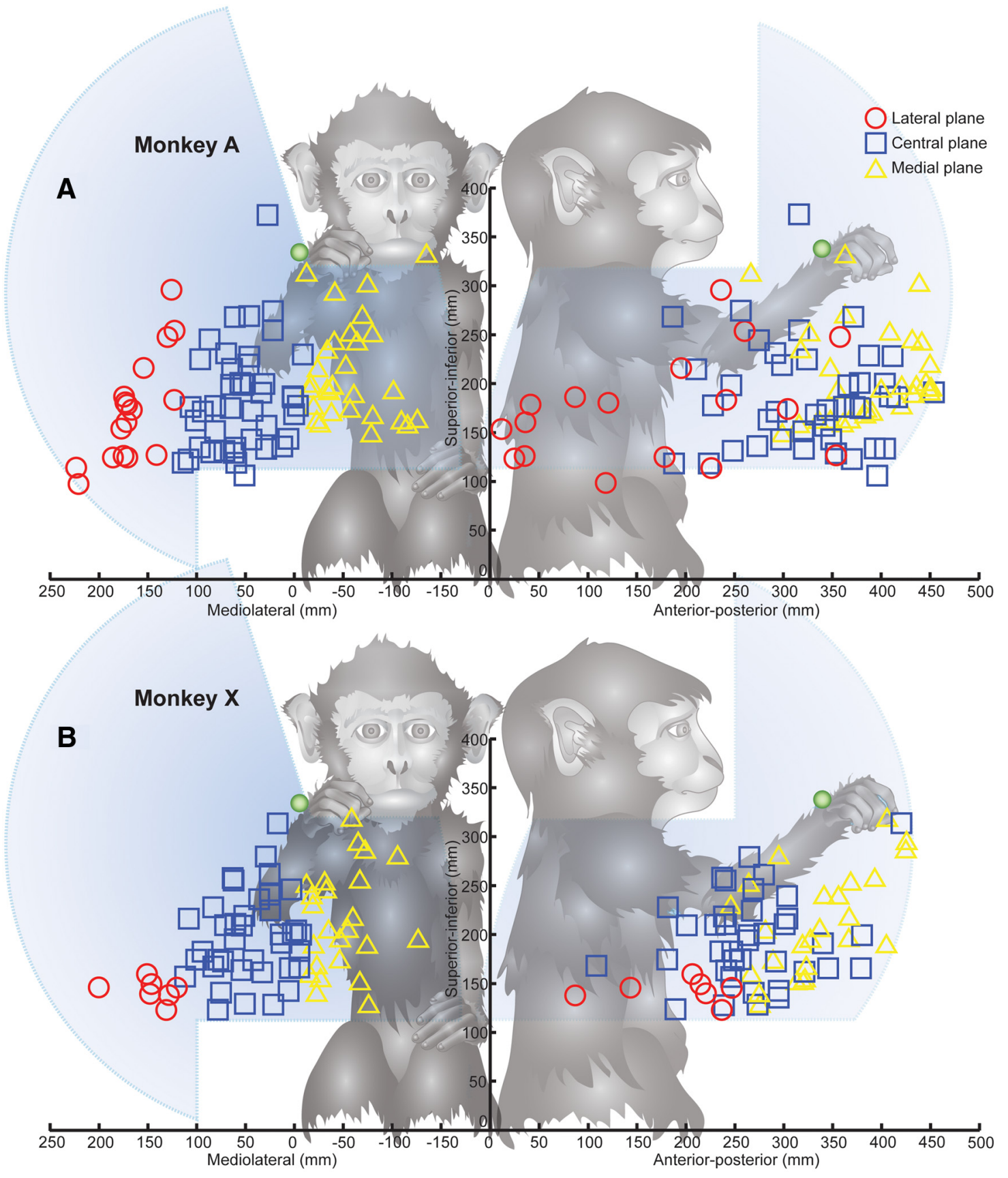

Figure 5. HFLD-ICMS-evoked forelimb movement spatial endpoints. $2 D$ mediolateral and anterior-posterior views of Monkey A's $(\boldsymbol{A})$ and Monkey X's $(\boldsymbol{B})$ workspace, including all HFLD-ICMS-evoked spatial endpoints for each monkey superimposed. Spatial endpoints are represented by colored shapes corresponding to mediolateral planes in which spatial endpoints reside (assigned in key legend). Lateral plane lies between the mediolateral coordinates of 116.1 and $242.2 \mathrm{~mm}$; central plane -10 to $116.1 \mathrm{~mm}$; medial plane -136.1 to -10 $\mathrm{mm}$. Each monkey's midsagittal plane is situated at approximately mediolateral coordinate $-100 \mathrm{~mm}$, and mid-coronal plane at approximately anterior-posterior $100 \mathrm{~mm}$. Green circle represents dorsal tubercle of radius. $\boldsymbol{A}, \boldsymbol{B}$, Large light blue sphere section represents an estimate of the workspace through which the dorsal tubercle of the radius was physically capable of moving.

stimulus site spacing) would correspond to $30-60 \mathrm{~mm}$ in the workspace. Of course, this number would actually be less because some of the representation is devoted specifically to distal movements. Nevertheless, the number is clearly much less than the actual distance obtained from our workspace maps.

That the mean 3D distance is less than if the relationship were random, yet greater than if the relationship were completely orderly and uniform, is consistent with the color-coded cortical representation maps of Figure $4 A, B$. Although there are many examples of adjacent sites yielding similar endpoints, such as the purple color-coded cortical sites surrounding site 47 (Fig. $4 A$, red outline), site 47 itself yielded a disparate endpoint, resulting in all adjacent sites relative to 47 having mean distances that were elevated. This can be seen when looking at the mean distance at the same site in Figure 6A (red outline). Another possible contributor is that similar spatial endpoints were often represented at multiple locations throughout M1, thereby lowering the mean distance for all possible site combination permutations. This is illustrated clearly by the color-coded patchwork representation of spatial endpoint maps in Figure $4 A, B$, revealing multiple cor- 
tical foci yielding similar endpoints. As a result, when assessing the M1 forelimb cortex as a whole, there is only a weak (but significant) correlation between increasing cortical site distance and the associated 3D spatial endpoint separation for both monkeys ( $r=0.0214, p=0.0158$; Pearson linear correlation). Nevertheless, although cortical site aggregates that yielded similar spatial endpoints often shared a border with cortical site aggregates yielding a disparate set of endpoints, a spatial endpoint continuity trend is largely present within aggregates throughout the cortex.

\section{Comparison of spatial endpoint maps across monkeys}

Although there were largely distinct cortical topographical differences in endpoint representations between monkeys, there were also similarities, such as hand-tomouth postures elicited from cortical loci neighboring the $\mathrm{M} 1$ face representation in both monkeys. This particular movement has been noted in previous studies evoking movement from the precentral cortex (Ferrier, 1875; Graziano and Aflalo, 2007). One example of similarly located cortical sites in each monkey yielding similar endpoint postures is cortical site 140 in Monkey A and cortical site 91 in Monkey X. Both cortical sites are near the M1 face representation (Fig. $1 A, B$, face muscle facilitation) on the convexity of the central sulcus, and both yielded endpoint postures with the monkey's hand near the mouth.

Figure 7 illustrates the spatial trajectories and endpoints resulting from stimulation at both sites, as well as the associated EMG activity for 23 forelimb muscles recorded during stimulation. Although not precisely identical, the pattern of spatial trajectory and of EMG activity (red tracings for Monkey A, blue for Monkey X) that yielded similar endpoint postures is quite similar across monkeys. Of particular note is that stimulation-evoked EMG activity is heavily tonic in nature with synchronous coactivation of antagonist muscles driving the arm to a final endpoint equilibrium posture (e.g., Fig. 7, elbow muscles). Also noteworthy is the difference between the tonic features of stimulus-evoked EMG activity (red traces) compared with Monkey A's voluntary EMG activity (black traces), both over similar movement trajectories from the food well to the mouth. Contrary to the coactivation of antagonist muscles in a predominantly tonic pattern throughout the stimulus-evoked movement, the muscle activity that occurred during voluntary movement over a very similar trajectory displays largely phasic activation of agonist and antagonist muscles
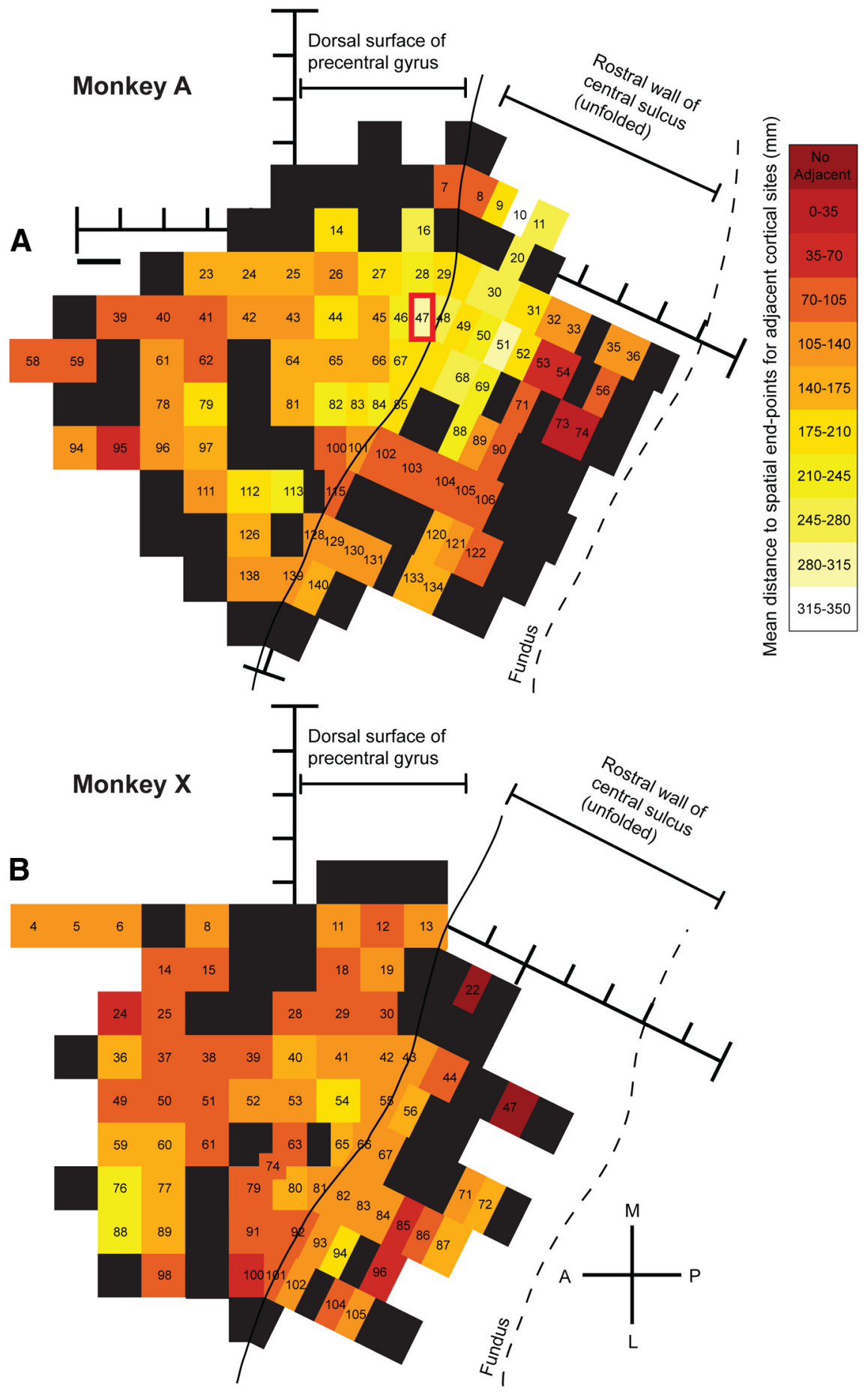

Figure 6. Maps representing the mean 3D Euclidean distance from the movement endpoint elicited from each cortical site to the spatial endpoints elicited from adjacent cortical sites. Adjacent cortical sites where stimulation failed to evoke stable spatial endpoints were excluded from this analysis. Maps are shown for Monkey A $(\boldsymbol{A})$ and Monkey $X(\boldsymbol{B})$. Adjacent cortical sites are those within $1 \mathrm{~mm}$ of each other. Each cortical stimulation site is numbered as in Figure 1. Color-coded mean distance key is illustrated. For Monkey $X$, cortical sites 22 and 47 have no adjacent cortical sites to which comparisons can be made and therefore are indicated by the color labeled "No Adjacent." The rostral wall of the central sulcus is unfolded into the same $2 \mathrm{D}$ plane as the dorsal surface of the precentral gyrus. Compass rose represents anterior, posterior, medial, and lateral. Scale bar, $1 \mathrm{~mm}$.

at varied time points during the movement. These results suggest that, although stimulus-evoked movements may sometimes appear grossly similar to natural movements, they are rather the result of muscle activation patterns that are quite different from voluntary movement over approximately the same trajectory. 


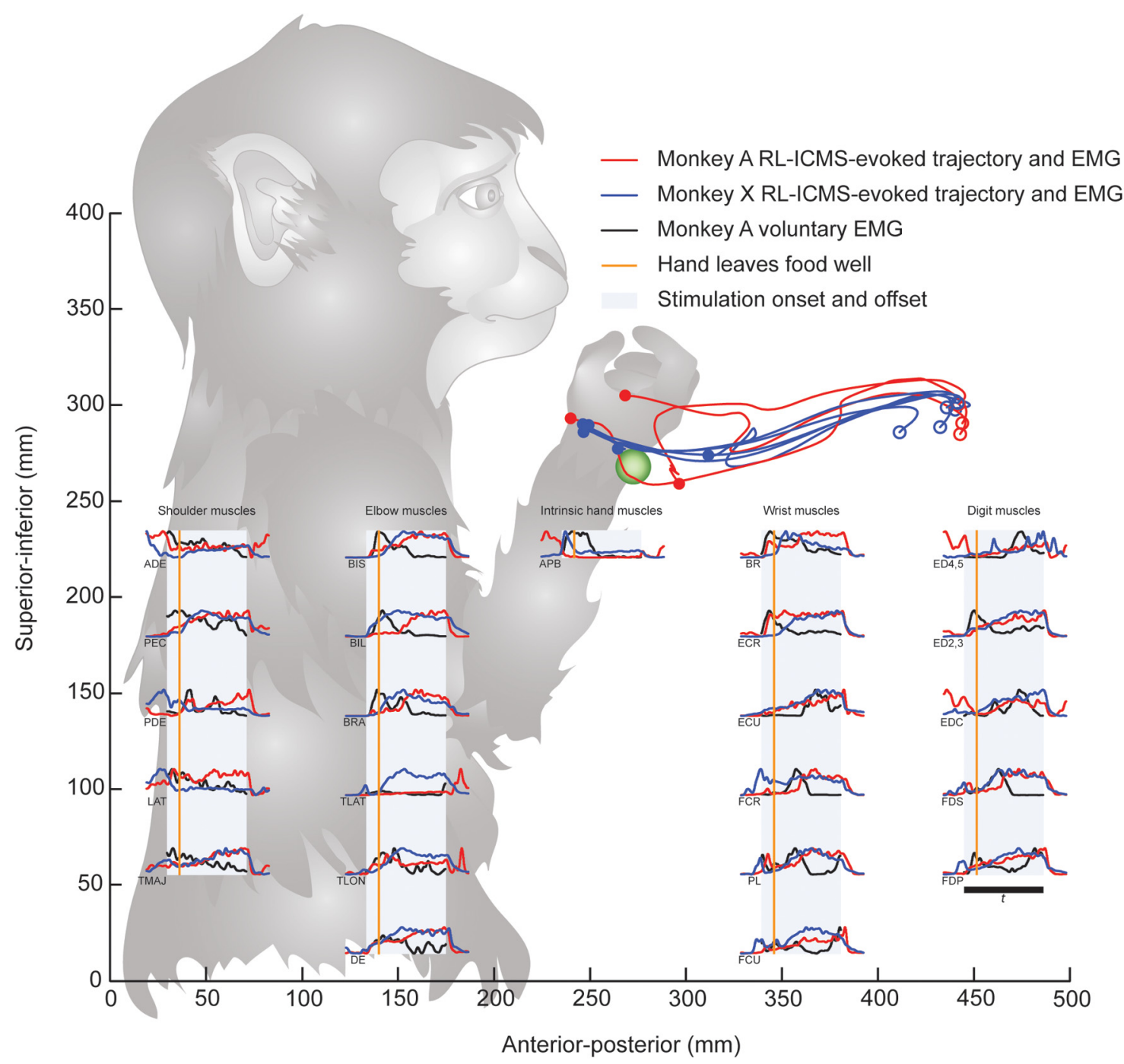

Figure 7. HFLD-ICMS-evoked EMG activity and trajectories across monkeys and compared with voluntary movement. EMG activity corresponding to movement trajectories and endpoints were recorded during stimulation of two similarly located M1 sites in each monkey (site 140 in Monkey A; site 91 in Monkey X) producing similar movement trajectories of the wrist marker (green sphere) through space from the food well to the mouth (red represents Monkey A; blue represents Monkey X). Stimulation was initiated at the food well for each trajectory trace (open circles) and ended near the mouth (solid circles). Mean stimulus-evoked spatial endpoints calculated for each monkey have a spatial separation from one another of $11.5 \mathrm{~mm}$. Red and blue EMG traces represent stimulus-evoked activity in Monkey A and Monkey X, respectively. Black EMG records correspond to voluntary activity in Monkey A over similar movement trajectories, from food well to mouth, as those elicited with stimulation. Start of stimulus-evoked trajectories and EMG correspond to stimulus initiation at the food well, and start of voluntary EMG corresponds to the hand leaving the food well signaled by an infrared beam present in the well. Voluntary EMG and stimulated EMG are aligned to correspond with the hand leaving the food well, which is represented by the orange vertical line in the EMG traces. Stimulus onset and offset, as well as duration, are indicated by solid light blue background. Each EMG record is averaged, rectified, and smoothed over the trials tested. Scale bar ( $t$ ) represents stimulation epoch of $1000 \mathrm{~ms}$. Green sphere represents dorsal tubercle of radius. Five shoulder muscles: PEC, Pectoralis major; ADE, anterior deltoid; PDE, posterior deltoid; TMAJ, teres major; LAT, latissimus dorsi. Seven elbow muscles: BIS, biceps short head; BIL, biceps long head; BRA, brachialis; BR, brachioradialis; TLON, triceps long head; TLAT, triceps lateral head; DE, dorsoepitrochlearis. Five wrist muscles: ECR, extensor carpi radialis; ECU, extensor carpi ulnaris; FCR, flexor carpi radialis; FCU, flexor carpi ulnaris; PL, palmaris longus. Five digit muscles: EDC, extensor digitorum communis; ED23, extensor digitorum 2 and 3; ED45, extensor digitorum 4 and 5; FDS, flexor digitorum superficialis; FDP, flexor digitorum profundus. One intrinsic hand muscle: APB, abductor pollicis brevis.

\section{Discussion}

The present study expands upon prior HFLD-ICMS studies by providing a comprehensive and systematically determined M1 map of forelimb movement endpoint representation. We used stimulus parameters determined in our laboratory (Van Acker et al., 2013) to be optimal for evoking forelimb movements to consistent and stable spatial endpoints in awake monkeys. Our mapping procedure encompassed the full extent of M1 cortex. The corresponding stimulus-evoked movements and endpoint positions constitute a relatively large, although incomplete, representation of the monkey's available movement space. In particular, movements to the most peripheral parts of the workspace were limited, and there were no movements above head level. Collectively, the present data suggest a broad topographical arrangement of movements elicited in $3 \mathrm{D}$ space that is represented in a patchwork fashion across the unfolded 2D M1 cortex. An important feature of these maps is that aggregates of adjacent sites often yield contiguous spatial endpoints, although disparate spatial endpoints from adjacent cortical sites were also common. Although lacking a clear orderly representation of the periphery, our maps of endpoint positions in M1 were not random. Analysis of adjacent cortical sites in terms of the physical distance separating the endpoints produced by stimulating each site showed that the average distance for all 
sites was significantly less than the mean distance obtained by calculating the distance between each endpoint position relative to every other endpoint position and the mean distance obtained by randomizing cortical site pairs. This shows that the organization of cortical output relative to stimulus-evoked endpoint positions is not random but rather has some degree of order in which adjacent cortical sites tend to have endpoint positions closer together than would be expected if the organization were random.

This raises an interesting question of what a highly ordered cortical map representing the 3D workspace of the arm might look like. The representation of space in the superior colliculus serves as an example of an orderly mapping of visual space directly on the 2D layered structure of the superior colliculus. However, this is not directly comparable because the direction of gaze in the visual field does not have depth and maps easily as a $2 \mathrm{D}$ array. Adding depth to a simple $2 \mathrm{D}$ representation of the workspace theoretically might be accomplished in different ways, for example, the firing rate of cells at a particular location. Stimulation at a given cortical site would then produce a movement in a particular direction in the workspace, and the distance traveled in that direction would be dependent on the stimulus frequency affecting the firing rate of neurons. Although this would be orderly and attractive in many ways, it does not correspond to what we observed. Our data are inconsistent with a $2 \mathrm{D}$ workspace map, and stimulating at different frequencies (within limits) always produced trajectories to nearly the same endpoint (Van Acker et al., 2013). Other possible models of representing the $3 \mathrm{D}$ workspace in a highly orderly way across motor cortex become even more contrived and nonphysiological. It is clear that the workspace itself is not represented in any highly orderly fashion across primary motor cortex. Prior discussion on this subject pointed out the difficulty and complexity that results from trying to represent in an orderly way a multidimensional space on a $2 \mathrm{D}$ cortical sheet (Graziano and Aflalo, 2007).

We observed by visual qualitative assessment that cortical sites yielding exclusively proximal or exclusively distal muscle facilitation in StTAs were less likely to yield complete movements to stable spatial endpoints. HFLD-ICMS-evoked activation of exclusively proximal or distal muscles presented the possibility that the unaffected muscles and associated joints could be voluntarily activated by the monkey during stimulation, thereby allowing movement of the wrist endpoint throughout the duration of stimulation. Although problematic for identifying stable endpoints, the fact that the monkey retained the ability to voluntarily control muscles at joints unaffected by HFLD-ICMS is relevant to understanding the extent of disruption of the internal motor program by repetitive stimulation. In addition to disrupting the execution steps of the motor program for those muscles affected directly by stimulation, it is also possible that aspects of the internal motor program for the whole limb movement may have become disrupted through antidromic and orthodromic activation of premotor cortical areas, subcortical areas, and spinal levels involved in motor planning and execution. Were this the case, HFLD-ICMS would have affected movements at joints beyond those whose muscles were affected directly by stimulation. However, our results show that this was not the case. The monkey retained voluntary control of muscles that were not directly affected by HFLD-ICMS, including muscles acting on joints adjacent to those in which muscle activity was completely stimulus-driven (Griffin et al., 2011).
The absence of movement endpoints about the head as well as to more extreme positions of the workspace is noteworthy. Although one possible explanation for not observing movements of the hand elevated above head level is that the force of gravity exerts a downward force on the stimulus-evoked movement of the arm, gravity cannot be the sole reason for the absence of such elevated movements in this study. We routinely evoked converging forelimb endpoint postures from initial positions above and below the final endpoint, corroborating previous evidence that final endpoint posture is consistent regardless of starting position of the limb (Graziano et al., 2005), provided that stimulation produced a sufficiently forceful activation of the muscle synergy represented at a particular cortical site. If gravity can be overcome through stimulus-evoked forelimb elevation from an inferior starting position to a position at or below head level, elevation above head level should be equally achievable were gravity the sole impediment. Rather than gravity, we attribute the lack of movement endpoints above the head and to other extremes of the workspace to coactivation of antagonist muscles, which prevented the full range of motion, particularly at shoulder and elbow joints.

Although similarities in the spatial endpoint maps of the two monkeys were limited, a few specific examples of similarity are notable. The spatial endpoint positions of the forelimb elicited from cortical sites bordering the face and forelimb representations tended to drive the monkey's hand near the mouth, a finding that has been described previously (Ferrier, 1875; Graziano and Aflalo, 2007). That this consistent finding was observed in the present study is less notable than how varied its interpretation can be. In part, because muscles of both the face and the arm are activated in concert during stimulation, this finding has been proposed previously to represent activation of an underlying physiologically relevant movement circuit yielding a feeding posture (Graziano and Aflalo, 2007). Alternatively, the movement could represent stimulus activation of efferent corticospinal output from the proximate arm and facial muscle representations, which can be seen in Figure 1. Were stimulation activating an underlying movement-specific circuit, each stimulus-evoked movement should mirror a movement that is useful for normal functioning and activity. In this case, the feeding motion would exhibit elbow flexion and wrist supination to bring the fingers to the mouth in a feeding motion, which is indeed what has been reported in previous studies (Ferrier, 1875; Graziano et al., 2002). However, were stimulation activating largely corticospinal projections within the proximity of the stimulating electrode as well as those activated trans-synaptically, such as near the convergence of face and forelimb representations, any number of movement combinations involving the face and forelimb might be elicited. $\mathrm{Al}-$ though stimulation of a few sites near the border of the forelimb and face representations in the present study elicited elbow flexion combined with wrist supination (Fig. 7), numerous other combinations of movements were also elicited near this area, leading the wrist to various spatial endpoint positions and postures. These include instances where flexion of the elbow was combined with pronation of the wrist, resulting in unnatural postures. This suggests that, although stimulus-evoked movements can appear natural and similar to voluntary movements, stimulation can also yield forelimb movements that appear to have no underlying purpose. These results are consistent with the hypothesis that stimulus-evoked endpoint positions and postures are produced through the activation of corticospinal outputs that converge downstream on motoneurons and spinal interneurons to yield forelimb movement equilibrium 
positions brought about through the length-tension relationships of activated muscles (Griffin et al., 2011; Cheney et al., 2013).

A previous hypothesis proposed that cortical stimulation can lead to target muscle activity switching from facilitation to suppression with opposing limb starting positions, resulting from stimulation activating natural underlying circuits, which have specific limb endpoints (Graziano et al., 2004). Were this the case, EMG activity recorded during stimulated movements should closely resemble the EMG activity of the same muscles during voluntary movements over the same trajectories. An alternative hypothesis is that stimulation activates neural elements through physical and physiological current spread to the tissue surrounding the stimulating electrode. The stimulus-evoked movement might therefore mimic natural movement through tonic activation of target muscles, and the forelimb's endpoint would then be a product of the summed length-tension relationships of the muscles. In this case, the EMG activity during voluntary movements would not be expected to match that of stimulated movements over the same trajectories.

Data from our laboratory support the latter hypothesis. Prior work in our laboratory suggests that switching of muscle activity from facilitation to suppression upon opposing limb starting positions rarely occurs (Griffin et al., 2011; Cheney et al., 2013). Instead, voluntary EMG activity is completely replaced with stimulus-evoked EMG activity, which can either raise or lower the existing level of activity. Additionally, it is clear that stimulus-evoked EMG activity shows tonic coactivation of antagonist muscles in a manner inconsistent with EMG patterns during voluntary movements over similar trajectories. The pattern of stimulus-evoked EMG activity suggests that stimulus-evoked movements and endpoint postures are the product of sustained activation of muscles that lead the forelimb to a physical equilibrium position. The fact that the majority of evoked movement endpoints in this study reside in the central portion of the workspace is consistent with the hypothesis that applying HFLD-ICMS to a single site in the cortex tends to activate colocalized flexor- and extensorspecific corticospinal elements, often resulting in activation of muscle ensembles that include antagonist muscles, which drive the arm to an intermediate equilibrium position. As pointed out above, this may explain why movements of the arm to positions above head level and to other extremes of the workspace were not found in this study, even though such movements can easily be made under voluntary control. Coactivation of antagonist muscles impeded the action of agonist muscles and limited the range of motion.

We would like to emphasize that the maps of cortical stimulation-mediated endpoint positions in the present study represent the full extent of spatial endpoints that could be elicited by systematically applying large-parameter HFLDICMS to individual sites throughout the M1 forelimb representation. Although it is likely that applying such stimulation parameters through two or more electrodes at multiple sites could elicit spatial endpoints equivalent to a vector sum of the individual spatial endpoints (Ethier et al., 2006), it is unlikely that endpoints more peripheral than those achieved with single-site stimulation could be achieved using this method. Clearly, the internal motor program activates output modules in motor cortex with more specificity than is achieved with HFLD-ICMS at the levels needed to produce complete movements to stable endpoints.
Our results do not rule out the existence of strong corticocortical connections that are often coactivated during recruitment of muscles to produce coordinated movements; however, they do not suggest that applying HFLD-ICMS to a single M1 cortical site selectively activates an individual physiologically relevant movement circuit, matching what occurs during voluntary movement. Our findings suggest that M1 neural encoding of movement capitalizes on strong corticospinal output with concomitant horizontal collateral connections to associated corticospinal outputs, both of which are activated with HFLDICMS provided sufficient stimulation parameters are applied. HFLD-ICMS thereby produces activation of motoneuron pools and subsequently muscle ensembles that result from cortical neuronal activation through both physical and physiological spread of stimulation.

\section{References}

Andriacchi TP, Alexander EJ, Toney MK, Dyrby C, Sum J (1998) A point cluster method for in vivo motion analysis: applied to a study of knee kinematics. J Biomech Eng 120:743-749. CrossRef Medline

Asanuma H, Rosén I (1972) Topographical organization of cortical efferent zones projecting to distal forelimb muscles in the monkey. Exp Brain Res 14:243-256. CrossRef Medline

Asanuma H, Sakata H (1967) Functional organization of a cortical efferent system examined with focal depth stimulation in cats. J Neurophysiol 30:35-54.

Belhaj-Saif A, Karrer JH, Cheney PD (1998) Distribution and characteristics of poststimulus effects in proximal and distal forelimb muscles from red nucleus in the monkey. J Neurophysiol 79:1777-1789. Medline

Buys EJ, Lemon RN, Mantel GW, Muir RB (1986) Selective facilitation of different hand muscles by single corticospinal neurones in the conscious monkey. J Physiol 381:529-549. Medline

Cheney PD, Fetz EE (1980) Functional classes of primate corticomotoneuronal cells and their relation to active force. J Neurophysiol 44:773-791. Medline

Cheney PD, Fetz EE (1985) Comparable patterns of muscle facilitation evoked by individual corticomotoneuronal (CM) cells and by single intracortical microstimuli in primates: evidence for functional groups of CM cells. J Neurophysiol 53:786-804. Medline

Cheney PD, Griffin DM, Van Acker GM 3rd (2013) Neural hijacking: action of high-frequency electrical stimulation on cortical circuits. Neuroscientist 19:434-441. CrossRef Medline

Ethier C, Brizzi L, Darling WG, Capaday C (2006) Linear summation of cat motor cortex outputs. J Neurosci 26:5574-5581. CrossRef Medline

Ferrier D (1875) Experiments on the brain of monkeys. Proc R Soc Lond 23:409-430.

Graziano MS, Aflalo TN (2007) Rethinking cortical organization: moving away from discrete areas arranged in hierarchies. Neuroscientist 13:138147. CrossRef Medline

Graziano MS, Taylor CS, Moore T (2002) Complex movements evoked by microstimulation of precentral cortex. Neuron 34:841-851. CrossRef Medline

Graziano MS, Patel KT, Taylor CS (2004) Mapping from motor cortex to biceps and triceps altered by elbow angle. J Neurophysiol 92:395-407. CrossRef Medline

Graziano MS, Aflalo TN, Cooke DF (2005) Arm movements evoked by electrical stimulation in the motor cortex of monkeys. J Neurophysiol 94: 4209-4223. CrossRef Medline

Griffin DM, Hudson HM, Belhaj-Saïf A, Cheney PD (2011) Hijacking cortical motor output with repetitive microstimulation. J Neurosci 31: 13088-13096. CrossRef Medline

Jankowska E, Padel Y, Tanaka R (1975) The mode of activation of pyramidal tract cells by intracortical stimuli. J Physiol 249:617-636. Medline

Lemon RN, Mantel GW, Muir RB (1986) Corticospinal facilitation of hand muscles during voluntary movement in the conscious monkey. J Physiol 381:497-527. Medline

McKiernan BJ, Marcario JK, Karrer JH, Cheney PD (1998) Corticomotoneuronal postspike effects in shoulder, elbow, wrist, digit, and intrinsic hand muscles during a reach and prehension task. J Neurophysiol 80: 1961-1980. Medline 
McKiernan BJ, Marcario JK, Karrer JH, Cheney PD (2000) Correlations between corticomotoneuronal (CM) cell postspike effects and cell-target muscle covariation. J Neurophysiol 83:99-115. Medline

Park MC, Belhaj-Saif A, Cheney PD (2000) Chronic recording of EMG activity from large numbers of forelimb muscles in awake macaque monkeys. J Neurosci Methods 96:153-160. CrossRef Medline

Park MC, Belhaj-Saiff A, Gordon M, Cheney PD (2001) Consistent features in the forelimb representation of primary motor cortex in rhesus macaques. J Neurosci 21:2784-2792. Medline

Park MC, Belhaj-Saï A, Cheney PD (2004) Properties of primary motor cortex output to forelimb muscles in rhesus macaques. J Neurophysiol 92:2968-2984. CrossRef Medline
Senesh M, Wolf A (2009) Motion estimation using point cluster method and Kalman filter. J Biomech Eng 131:051008. CrossRef Medline

Stepniewska I, Preuss TM, Kaas JH (2006) Ipsilateral cortical connections of dorsal and ventral premotor areas in New World owl monkeys. J Comp Neurol 495:691-708. CrossRef Medline

Tehovnik EJ, Tolias AS, Sultan F, Slocum WM, Logothetis NK (2006) Direct and indirect activation of cortical neurons by electrical microstimulation. J Neurophysiol 96:512-521. CrossRef Medline

Van Acker GM 3rd, Amundsen SL, Messamore WG, Zhang HY, Luchies CW, Kovac A, Cheney PD (2013) Effective intracortical microstimulation parameters for evoking forelimb movements to stable spatial end-points from primary motor cortex. J Neurophysiol. 